\title{
Further Geometric Restrictions on Jordan Structure in Matrix Factorization *
}

\author{
Charles R. Johnson ${ }^{1}$, Drew Lewis ${ }^{2}$, Yulin Zhang ${ }^{3 \dagger}$ \\ ${ }^{1}$ Department of Mathematics, College of William and Mary, Williamsburg, VA, 23187 \\ crjohnso@math.um.edu \\ ${ }^{2}$ Department of Mathematics, University of Alabama \\ andrew.m.lewis@ua.edu \\ ${ }^{3}$ Centro de Matemática, Universidade do Minho, 4710 Braga, Portugal \\ zhang@math.uminho.pt
}

October 10, 2011

\begin{abstract}
It is known that a nonsingular, nonscalar, $n$-by- $n$ complex matrix $A$ may be factored as $A=B C$, in which the spectra of $\mathrm{B}$ and $\mathrm{C}$ are arbitrary, subject to $\operatorname{det}(A)=\operatorname{det}(B) \operatorname{det}(C)$. Furthermore, it is also known that $\mathrm{B}$ and $\mathrm{C}$ can be taken to be nonderogatory. Additionally it has been shown that when two matrices have eigenvalues of high geometric multiplicity, this restricts the possible Jordan structure of the third. We demonstrate a previously unknown restriction on the Jordan structures of B and C. Furthermore, we show that this generalized geometric multiplicity restriction implies the already known geometric multiplicity restriction, show that the new condition is more restrictive, is not sufficient in general but is sufficient in a situation that we identify.
\end{abstract}

Keywords: Jordan form; geometric multiplicity; matrix product.

AMS Classifications: 15A23

\section{Introduction}

In [15] it was shown that any nonsingular nonscalar matrix $A \in M_{n}(\mathbb{C})$ may be factored $A=B C$, so that $B, C \in M_{n}(\mathbb{C})$ have arbitrary spectra, subject only to the obvious determinantal condition $\operatorname{det} A=\prod_{i=1}^{n} \beta_{i} \prod_{i=1}^{n} \gamma_{i}$, in which $\beta_{1}, \beta_{2}, \ldots, \beta_{n}$ are the eigenvalues of $B$ and $\gamma_{1}, \gamma_{2}, \ldots, \gamma_{n}$ are the eigenvalues of $C$ (repeats allowed). In [6] Sourour's theorem was extended to the case where exactly $n-\operatorname{rank} A$ of the elements $\beta_{1}, \beta_{2}, \ldots, \beta_{n}, \gamma_{1}, \gamma_{2}, \ldots, \gamma_{n}$ are equal to zero. If $B$ and $C$ have repeated eigenvalues, no indication is given in [15] what sort of

${ }^{*}$ This study was supported in part by National Science Foundation Grant DMS-03-53510,USA, CMAT through the FCT Pluriannual Funding Program and PTDC/MAT/112273/2009, Portugal.

${ }^{\dagger}$ Corresponding author 
Jordan structure they may have, and unfortunately, the proof there is not easily adapted to further specify the Jordan structure.

The Jordan structure of $B$ and $C$ cannot generally be taken to be arbitrary. (Suppose that $B$ and/or $C$ have repeated eigenvalues and 1-by-1 Jordan blocks in case $n=2$, for example.) Thus a natural, deeper, question is to ask for a given $A$ and specified eigenvalues $\beta_{1}, \beta_{2}, \ldots, \beta_{n}$ for $B$ and $\gamma_{1}, \gamma_{2}, \ldots, \gamma_{n}$ for $C$, $\prod_{i=1}^{n} \beta_{i} \gamma_{i}=\operatorname{det} A$, what the Jordan form for $B$ and $C$ may be?

This is known to be a very difficult problem, in general, but a number of prior works discuss portions of it, some explicitly and some very theoretically. For example, it was shown in [9], [11] that, over the complex field, B and C may be taken to be nonderogatory, for any allowed spectra specified for them. In [1] this result was generized over any field with at least four elements. And [7], [8] showed that the geometric multiplicity restrictions together with the obvious determinantal condition on the eigenvalues, are necessary and sufficient for the problem when possible Jordan forms are given for $n<4$, but not for $n \geq 4$. Paper [10] considered the possible Jordan canonical forms of $A, B, C$ when they are diagonalizable with two distinct eigenvalues, and [5] gives a criterion for writing a given square matrix as the product of two matrices with prescribed spectra and ranks, etc. A number of variations have been studied in the literatures in the last 20 years; see [13], [14], [17] and references therein.

We must mention that the above factorization problem falls under a more general problem mentioned in the literature in recent years: Given similarity classes $C_{1}, \ldots C_{k}$, determine whether or not the equation $A_{1} \cdots A_{k}=I$ can be solved with $A_{i} \in C_{i}$. A weaker version of this problem was solved by CrawleyBoevey [3], [4] over the complex field in which the matrices $A_{i}$ belong to the closures of the similarity classes $C_{i}$. Finally, we adopt the convention that, in a given matrix, the use of a different variable will denote a distinct eigenvalue unless otherwise stated.

\section{Observations}

We begin with some useful observations. One is the fact that our problem is symmetric in the three matrices $A, B$ and $C$. Since all three matrices must be invertible, we may also write, for example,

$$
B=A C^{-1} \text {. }
$$

Since the Jordan structure of $C^{-1}$ is determined by that of $C$, and since $A C^{-1}$ is similar to $C^{-1} A$, it is equivalent to take any of the three matrices as the left hand side and the other two matrices in either order.

Another is that the Jordan structure is similarity invariant, and similarity may be passed through the equation (e.g. $S^{-1} A S=S^{-1} B S S^{-1} C S$ ). We may take, without loss of generality, any one of the three matrices to actually be in Jordan structure.

Throughout all this work, we use $g_{T}(\lambda)$ denote the geometric multiplicity of the eigenvalue $\lambda$ in the matrix $T$. We use $m_{T}(\lambda)$ to denote the algebraic 
multiplicity of $\lambda$ in the matrix $\mathrm{T}$.

\section{The Generalized Geometric Multiplicity Restric- tion}

Here, extending the work of [2] and [16], the geometric multiplicity restriction (GMR) of [8] is properly generalized. The new GGMR is still not sufficient, as we show, but it is quite explicit; and we show that it is sufficient in a restricted setting.

Theorem 1 (Generalized Geometric Multiplicity Restriction(GGMR)) There exist nonsingular matrices $A, B, C \in M_{n}(\mathbb{C})$ with prescribed invariant factors $h_{1}(A)\left|h_{2}(A)\right| \cdots\left|h_{n}(A), h_{1}(B)\right| h_{2}(B)|\cdots| h_{n}(B)$ and $h_{1}(C)\left|h_{2}(C)\right| \cdots \mid h_{n}(C)$, respectively, such that $A=B C$ only if $\operatorname{det}(A)=\operatorname{det}(B) \operatorname{det}(C)$ and the following are satisfied for all $\alpha \in(A), \beta \in(B)$ and $\gamma \in(C)$ :
1. $h_{i}\left(\alpha C^{-1}\right) \mid h_{n-g_{A}(\alpha)+i}(B)$ for $i=1, \ldots, g_{A}(\alpha)$
2. $h_{i}\left(\alpha B^{-1}\right) \mid h_{n-g_{A}(\alpha)+i}(C)$ for $i=1, \ldots, g_{A}(\alpha)$
3. $h_{j}(\beta C) \mid h_{n-g_{B}(\beta)+j}(A) \quad$ for $j=1, \ldots, g_{B}(\beta)$
4. $h_{j}\left(\frac{1}{\beta} A\right) \mid h_{n-g_{B}(\beta)+j}(C) \quad$ for $j=1, \ldots, g_{B}(\beta)$
5. $h_{k}\left(\frac{1}{\gamma} A\right) \mid h_{n-g_{C}(\gamma)+k}(B) \quad$ for $k=1, \ldots, g_{C}(\gamma)$
6. $h_{k}(\gamma B) \mid h_{n-g_{C}(\gamma)+k}(A) \quad$ for $k=1, \ldots, g_{C}(\gamma)$

Before we prove the theorem, we first present a previous result of Thompson [16]. The main result of his paper was to determine the possible values of the invariant factors of the matrix $A$ under rank one perturbations.

Theorem 2 [16] Let $A \in M_{n}(\mathbb{F})$ have similarity invariants $h_{1}(A)\left|h_{2}(A)\right| \cdots \mid h_{n}(A)$. Then, as column n-tuple $x$ and row $n$-tuple $y$ range over all vectors with entries in $\mathbb{F}$. The similarity invariants assumed by the matrix

$$
B=A+x y
$$

are precisely the monic polynomials $h_{1}(B)\left|h_{2}(B)\right| \cdots \mid h_{n}(B)$ for which

1. $\operatorname{deg}\left\{h_{1}(B) h_{2}(B) \cdots h_{n}(B)\right\}=n$

2. $h_{1}(B)\left|h_{2}(A)\right| h_{3}(B) \mid h_{4}(A) \cdots$

$$
h_{1}(A)\left|h_{2}(B)\right| h_{3}(A) \mid h_{4}(B) \cdots \text {. }
$$

Applying this theorem, we have the follow corollary which gives the interlacing relationships for algebraic multiplicity of eigenvalues of $A$ and $B$. 
Corollary 1 Under the same condition of theorem 2. Suppose that a is an eigenvalue of $A$, and $(x-a) \mid h_{i}(A)$, but $(x-a) \backslash h_{i-1}(A)$, then the algebraic multiplicity of a, satisfy the following interlacing inequality:

(i) $m_{i}(A) \leq m_{i+1}(B) \leq m_{i+2}(A) \leq m_{i+3}(B) \cdots$

(ii) $m_{i+1}(A) \leq m_{i+2}(B) \leq m_{i+3}(A) \cdots$.

where $m_{i}(A)$ (resp. $m_{i}(B)$ ) is the multiplicity of a as root of $h_{i}(A)$ (resp. $\left.h_{i}(B)\right)$.

Another corollary that comes directly from theorem 2 is that

Corollary 2 Assume that $D \in M_{n}(\mathbb{F})$ is a matrix under rank $r$ perturbations of $A$, then the invariant factors of $D, h_{1}(D)\left|h_{2}(D)\right| \cdots \mid h_{n}(D)$ and the invariant factors of $A$, satisfy the following interlacing relations

(1) $h_{1}(A)\left|h_{1+r}(D)\right| h_{1+2 r}(A) \mid h_{1+3 r}(D) \cdots$

(2) $h_{1}(D)\left|h_{1+r}(A)\right| h_{1+2 r}(D) \mid h_{1+3 r}(A) \cdots$

Suppose that $a$ is an eigenvalue of $A$, and $(x-a) \mid h_{i}(A)$, but $(x-a) \chi h_{i-1}(A)$, then the algebraic multiplicity of a, satisfy the following interlacing inequality:

(3) $m_{i}(A) \leq m_{i+r}(D) \leq m_{i+2 r}(A) \leq m_{i+3 r}(D) \cdots$

(4) $m_{i+r}(A) \leq m_{i+2 r}(D) \leq m_{i+3 r}(A) \cdots$.

In fact, the algebraic multiplicity of a root of $h_{i}(A)$ is exactly the Jordan block size associated with the eigenvalue of $A$. So, based on the above analysis, we may give an alternate statement of the theorem to facilitate its understanding. The reader can easily verify the equivalence.

Let nonsingular matrices $A, B, C \in M_{n}(\mathbb{C})$ have Jordan structures

$$
\begin{array}{ccc} 
& \text { eiganvalues } & \text { blocksizes } \\
A & \alpha_{1}: & a_{11} \leq \ldots \leq a_{1 p_{1}} \\
& \alpha_{2}: & a_{21} \leq \ldots \leq a_{2 p_{2}} \\
& \vdots & \\
& \alpha_{h}: & a_{h 1} \leq \ldots \leq a_{h p_{h}} \\
B & \beta_{i}: & b_{i 1} \leq \ldots \leq b_{i q_{i}}, \quad i=1, \ldots, k \\
C & \gamma_{i}: & c_{i 1} \leq \ldots \leq c_{i s_{i}}, \quad i=1, \ldots, l
\end{array}
$$

where $\alpha_{i}=\alpha_{j}, \beta_{i}=\beta_{j}, \gamma_{i}=\gamma_{j}$ only if $i=j$.

Theorem 3 (GGMR Restated) There exists a triple with the above Jordan structures and $A=B C$ only if $\operatorname{det}(A)=\operatorname{det}(B) \operatorname{det}(C)$ and 
(a) if $q_{i}+s_{j}>n$, then some $\alpha_{t}=\beta_{i} \gamma_{j}, p_{t} \geq q_{i}+s_{j}-n$, and

$$
\left(a_{t, p_{t}-\left(q_{i}+s_{j}-n\right)+1}, \ldots, a_{t, p_{t}}\right) \geq\left(b_{i 1}, \ldots, b_{i,\left(q_{i}+s_{j}-n\right)}\right),\left(c_{j 1}, \ldots, c_{j,\left(q_{i}+s_{j}-n\right)}\right)
$$

(b) if $q_{i}+p_{j}>n$, then some $\gamma_{t}=\frac{\alpha_{j}}{\beta_{i}}, s_{t} \geq q_{i}+p_{j}-n$, and

$$
\left(c_{t, s_{t}-\left(q_{i}+p_{j}-n\right)+1}, \ldots, c_{t, s_{t}}\right) \geq\left(b_{i 1}, \ldots, b_{i,\left(q_{i}+p_{j}-n\right)}\right),\left(a_{j 1}, \ldots, a_{j,\left(q_{i}+p_{j}-n\right)}\right)
$$

and

(c) if $p_{i}+s_{j}>n$, then some $\beta_{t}=\frac{\alpha_{i}}{\gamma_{j}}, q_{t} \geq p_{i}+s_{j}-n$, and

$$
\left(b_{t, q_{t}-\left(p_{i}+s_{j}-n\right)+1}, \ldots, b_{t, q_{t}}\right) \geq\left(a_{i 1}, \ldots, a_{i,\left(p_{i}+s_{j}-n\right)}\right),\left(c_{j 1}, \ldots, c_{j,\left(p_{i}+s_{j}-n\right)}\right) .
$$

Now we prove the theorem 1.

Proof. We will show that condition 3 in theorem 1 is necessary, and the proofs of the other five statements follow along the same lines. Let $\alpha \in$ $(A), \beta \in(B)$ and $\gamma \in(C)$ be given, and let $R=B-\beta I$. We then write $A=B C=(\beta I+R) C$ and we easily obtain

$$
A=\beta C+R C=\beta C+\sum_{i=1}^{b} R_{i},
$$

where $b=\operatorname{rank}(R C)=\operatorname{rank}(R)=\operatorname{rank}(B-\beta I)=n-g_{B}(\beta)$ and $\operatorname{rank}\left(R_{i}\right)=1$ for $i=1,2, \ldots, b$.

We can consider $A$ as a rank $b$ perturbation of $\beta C$. By corollary 2 , we have

$$
h_{t}(\beta C) \mid h_{t+b}(A), t=1,2, \ldots, g_{B}(\beta) .
$$

Since $b=n-g_{B}(\beta)$, we have shown 3 .

To show 6 , let $S=C-\gamma I$ and write $A=B(\gamma I+S)=\gamma B+B S$. Then, proceed along the same lines, substituting variable names as necessary.

For 1 and 5 , rewrite as $B=A C^{-1}$; we may then substitute $B, A, C^{-1}, \beta, \alpha$ and $\frac{1}{\gamma}$ for $A, B, C, \alpha, \beta$ and $\gamma$, respectively, into the above argument. The proof proceeds in the exact same manner. For 2 and 4, we can rewrite as $C=B^{-1} A$ and a similar substitution will generate a proof along the same lines.

We will now use the generalized geometric multiplicity restriction to give alternate proofs of the geometric multiplicity restriction from [7], [8].

Corollary 3 If $A, B, C \in M_{n}(\mathbb{C}), A$ is nonsingular and $A=B C$, then

(i) $g_{A}(\beta \gamma) \geq g_{B}(\beta)+g_{C}(\gamma)-n$ and

(ii) $g_{B}\left(\frac{\alpha}{\gamma}\right) \geq g_{A}(\alpha)+g_{C}(\gamma)-n$. 
Proof. Here we show only $(i),(i i)$ can be shown similarly. If $g_{B}(\beta)+$ $g_{C}(\gamma)-n \leq 0$, obviously, $g_{A}(\beta \gamma) \geq 0$. Thus we assume $g_{B}(\beta)+g_{C}(\gamma)-n>0$. We know that $(x-\gamma) \mid h_{n-g_{c}(\gamma)+1}(C)$, so $(x-\beta \gamma) \mid h_{n-g_{c}(\gamma)+1}(\beta C)$. Now, since $n-g_{C}(\gamma)<g_{B}(\beta)$, so $n-g_{C}(\gamma)+1 \leq g_{B}(\beta)$, by theorem 1 part 3 , we have that

$$
(x-\beta \gamma)\left|h_{n-g_{C}(\gamma)+1}(\beta C)\right| h_{\left(n-g_{B}(\beta)\right)+\left(n-g_{C}(\gamma)+1\right)}(A)=h_{n-\left(g_{B}(\beta)+g_{C}(\gamma)-n\right)+1}(A) .
$$

Thus, we have $g_{A}(\beta \gamma) \geq g_{B}(\beta)+g_{C}(\gamma)-n$.

The following example shows the generalized geometric multiplicity restriction(GGMR) is more general than geometric multiplicity restriction(GMR).

Example The follow triple

$$
A=\left[\begin{array}{llll}
1 & 0 & 0 & 0 \\
0 & 1 & 0 & 0 \\
0 & 0 & 1 & 0 \\
0 & 0 & 0 & 2
\end{array}\right], \quad B=\left[\begin{array}{llll}
1 & 1 & 0 & 0 \\
0 & 1 & 0 & 0 \\
0 & 0 & 1 & 1 \\
0 & 0 & 0 & 1
\end{array}\right] \text { and } C=\left[\begin{array}{cccc}
1 & 0 & 0 & 0 \\
0 & 1 & 0 & 0 \\
0 & 0 & 1 & 0 \\
0 & 0 & 0 & 2
\end{array}\right]
$$

satisfy the geometric multiplicity restriction and determinant restriction, but fail the generalized geometric multiplicity restriction.

Observation: In case that all $A, B, C$ are all diagonalizable, the generalized geometric multiplicity restriction and geometric multiplicity restriction is equivalent since in this case the algebraic and geometric multiplicities are equal. And we can use the same example which we showed in [8] as an example to say that theorem 1 is not sufficient.

\section{A Particular Sufficient Result}

Here we consider a special case and begin with an example. Suppose that $n=6$, the invariant factors of $B$ are $1,1,1,(x-\beta)^{2},(x-\beta)^{2},(x-\beta)^{2}$ and the invariant factors of $C$ are $1,(x-\gamma),(x-\gamma),(x-\gamma),(x-\gamma),(x-\gamma)(x-\delta), \gamma \neq \delta$, respectively. So $g_{B}(\beta)=3, g_{C}(\gamma)=5$. Necessary that there is an $A$ such that $A=B C$, we have that $g_{A}(\beta \gamma) \geq g_{B}(\beta)+g_{C}(\gamma)-n=3+5-6=2$ and the 6 interlacing conditions of theorem 1 must be verified. By item 6 of theorem 1 , $h_{5}(\gamma B) \mid h_{6}(A)$ and $h_{4}(\gamma B) \mid h_{5}(A)$, that is, $(x-\beta \gamma)^{2}$ must be a factor of $h_{5}(A)$ and of $h_{6}(A)$. Then, by condition 5 of theorem $1, h_{5}\left(\frac{1}{\gamma} A\right) \mid h_{6}(B)$, so $h_{5}(A)$ is exactly $(x-\beta \gamma)^{2}$. And since $h_{2}\left(\frac{1}{\gamma} A\right) \mid h_{3}(B)$, and $h_{3}(B)=1$, so $h_{2}(A)=1$, and $g_{A}(\beta \gamma)$ is at most 4 .

The possible invariant factors of $A$ are

(1) $1,1,(x-\beta \gamma),(x-\beta \gamma),(x-\beta \gamma)^{2},(x-\beta \gamma)^{2}$.

(2) $1,1,1,(x-\beta \gamma)^{2},(x-\beta \gamma)^{2},(x-\beta \gamma)^{2}$.

(3) $1,1,1,(x-\beta \gamma),(x-\beta \gamma)^{2},(x-\beta \gamma)^{2}(x-\beta \delta)$.

(4) $1,1,1,1,(x-\beta \gamma)^{2},(x-\beta \gamma)^{3}(x-\beta \delta)$. 
(5) $1,1,1,1,(x-\beta \gamma)^{2},(x-\beta \gamma)^{2}(x-d)^{2}, d^{2}=\beta^{2} \gamma \delta$.

(6) $1,1,1,1,(x-\beta \gamma)^{2},(x-\beta \gamma)^{2}(x-a)(x-b), \quad a \neq b, \quad a b=\beta^{2} \gamma \delta$.

But, by determinant restriction, the first and the second are ruled out, or $\gamma=\delta$, so only the other four remain. When (3) happens, let

$A$.

$B=\left[\begin{array}{ll}\beta & 1 \\ 0 & \beta\end{array}\right] \oplus\left[\begin{array}{ll}\beta & 1 \\ 0 & \beta\end{array}\right] \oplus\left[\begin{array}{ll}\beta & 1 \\ 0 & \beta\end{array}\right], C=\gamma I_{5} \oplus \delta$. Then, $B C$ gives directly

In case (4), it is easy to verify that there are

$B=\left[\begin{array}{ll}\beta & 1 \\ 0 & \beta\end{array}\right] \oplus\left[\begin{array}{ll}\beta & 1 \\ 0 & \beta\end{array}\right] \oplus\left[\begin{array}{ll}\beta & 1 \\ 0 & \beta\end{array}\right]$ and $C^{\prime}=\left[\begin{array}{llllll}\gamma & 0 & 0 & 0 & 0 & 0 \\ 0 & \gamma & 0 & 0 & 0 & 0 \\ 0 & 0 & \gamma & 0 & 0 & 0 \\ 0 & 0 & 0 & \gamma & 1 & 0 \\ 0 & 0 & 0 & 0 & \delta & 0 \\ 0 & 0 & 0 & 0 & 0 & \gamma\end{array}\right] \simeq C$

such that $B C^{\prime}$ has (4) as invariant factor.

In case (5) and (6), we write $B=\left[\begin{array}{cc}\beta & 1 \\ 0 & \beta\end{array}\right] \oplus\left[\begin{array}{ll}\beta & 1 \\ 0 & \beta\end{array}\right] \oplus\left[\begin{array}{ll}\beta & 1 \\ 0 & \beta\end{array}\right], C=$ $\gamma I_{4} \oplus\left[\begin{array}{ll}\gamma & 0 \\ 0 & \delta\end{array}\right]$ and $A=\left[\begin{array}{ll}\beta \gamma & 1 \\ 0 & \beta \gamma\end{array}\right] \oplus\left[\begin{array}{ll}\beta \gamma & 1 \\ 0 & \beta \gamma\end{array}\right] \oplus A^{\prime}$, where $A^{\prime}=\left[\begin{array}{ll}d & 1 \\ 0 & d\end{array}\right]$ or $\left[\begin{array}{ll}a & 0 \\ 0 & b\end{array}\right]$, since the last block of $B$ and of $C$ are nonderogatory, and $A^{\prime}$ is nonscalar, so by [9], there are $B^{\prime}$ similar to $B$ and $C^{\prime}$ similar to $C$, such that $B^{\prime} C^{\prime}$ with (5) or (6) as invariant factors.

In general, for this particular case, we have the following corollary which shows that in a particular situation the theorem 1 is necessary and sufficient.

Corollary 4 Suppose that $B=\left[\begin{array}{ll}\beta & 1 \\ 0 & \beta\end{array}\right] \oplus\left[\begin{array}{ll}\beta & 1 \\ 0 & \beta\end{array}\right] \oplus \cdots \oplus\left[\begin{array}{ll}\beta & 1 \\ 0 & \beta\end{array}\right], C=$ $\gamma I_{n-1} \oplus \delta$. Then the necessary conditions if theorem 1 are also sufficient for there exist $B^{\prime}$ similar to $B$ and $C^{\prime}$ similar to $C$, such that $B^{\prime} C^{\prime}$ lies in the similarity class of a given $A$.

Proof. Here we consider only the sufficiency. In this case, $g_{B}(\beta)=\frac{n}{2}$, $g_{C}(\gamma)=n-1$. So, $g_{A}(\beta \gamma) \geq g_{B}(\beta)+g_{C}(\gamma)-n=\frac{n}{2}+n-1-n=\frac{n}{2}-1$ must be satisfied. Assume that $A$ satisfies the 6 interlacing conditions in theorem 1. By 6,

$$
h_{i}(\gamma B) \mid h_{1+i}(A), \quad i=1, \ldots, n-1
$$

which means the invariant factors $h_{i}(A), i=\frac{n}{2}+2, \ldots, n$ must have $(x-\beta \gamma)^{2}$ as factors. By 5 ,

$$
h_{i}\left(\frac{1}{\gamma} A\right) \mid h_{1+i}(B), i=1, \ldots, n-1 .
$$

So the invariant factors $h_{i}(A), i=\frac{n}{2}+2, \ldots, n-1$ are exactly $(x-\beta \gamma)^{2}$. Then the possible invariant factors of $A$ are the follows 
(1) $\overbrace{1, \ldots, 1}^{\frac{n}{2}-1},(x-\beta \gamma),(x-\beta \gamma),(x-\beta \gamma)^{2}, \ldots,(x-\beta \gamma)^{2}$.

(2)

$\overbrace{1, \ldots, 1}^{\frac{n}{2}},(x-\beta \gamma)^{2},(x-\beta \gamma)^{2},(x-\beta \gamma)^{2}$.

(3) $\overbrace{1, \ldots, 1}^{\frac{n}{2}-1},(x-\beta \gamma),(x-\beta \gamma)^{2}, \ldots,(x-\beta \gamma)^{2},(x-\beta \gamma)^{2}(x-\beta \delta)$.

(4) $\overbrace{1, \ldots, 1}^{\frac{n}{2}+1},(x-\beta \gamma)^{2}, \ldots,(x-\beta \gamma)^{2},(x-\beta \gamma)^{3}(x-\beta \delta)$.

(5) $\overbrace{1, \ldots, 1}^{\frac{n}{2}+1},(x-\beta \gamma)^{2}, \ldots,(x-\beta \gamma)^{2},(x-\beta \gamma)^{2}(x-d)^{2}, d^{2}=\beta^{2} \gamma \delta$.

(6) $\overbrace{1, \ldots, 1}^{\frac{n}{2}+1},(x-\beta \gamma)^{2}, \ldots,(x-\beta \gamma)^{2},(x-\beta \gamma)^{2}(x-a)(x-b), \quad a \neq b, \quad a b=\beta^{2} \gamma \delta$.

Now, taking into account the determinant restriction, the cases (1) and (2) can not happen. In the other four cases, we can use the same arguments as we used in the $6 \times 6$ example to get $B^{\prime}$ similar to $B$ and $C^{\prime}$ similar to $C$, such that $B^{\prime} C^{\prime}$ lies in the similarity class of a given $A$.

\section{References}

[1] J.D. Botha, Spectrally arbitrary, nonderogatory factorization over a general field, Linear Algebra and Its Applications 433 (2010), 1-11.

[2] D. Carlson and E. Marques de Sá, Generalized minimax and interlacing theorem, Linear and Multilinear Algebra 15 (1984), 77-103.

[3] William Crawley-Boevey, Indecomposable parabolic bundles and the existence of matrices in prescribed conjugacy class closures with product equal to the identity, Publ. Math. Inst. Hautes Etudes Sci. 100 (2004), 171-207.

[4] William Crawley-Boevey, Quiver algebras, weighted projective lines, and the Deligne-Simpson problem, 'Proceedings of the International Congress of Mathematicians', vol. 2, Madrid 2006, eds M. Sanz-Solé et al. (European Mathematical Society, January 2007), 117-129.

[5] S. Furtado, L. Iglesias, F. C. Silva, Products of matrices with prescribed spectra and ranks, Linear Algebra and Its Applications 340 (2002) 137147.

[6] R. A. Horn and C. R. Johnson, Topics in Matrix Analysis, Cambridge Univ.Press, 1991. 
[7] C. R. Johnson and D. Lewis, Arbitrariness of Jordan Structure in Factorization, REU Report to the National Science Foundation, William and Mary, 2004.

[8] C. R. Johnson, D. Lewis and Yulin Zhang, Arbitrainess of Jordan Structure in Factorization: the Geometric Multiplicity Restriction and the 3-by-3 Case, submitted, 2011.

[9] C. R. Johnson and Yulin Zhang, Spectrally Arbitrary Factorization: The Nonderogatory Case. Textos de Matemática, Universidade de Coimbra, Série B, №39, 79-92, 2006.

[10] C. R. Johnson and Yulin Zhang, The Jordan Form Problem for $C=A B$ : the Balanced, Diagonalizable Case, Asian-European Journal of Mathematics, vol.3, No.4 (2010) 607-621.

[11] A. Lev, Product of Cyclic Similarity Classes in the Group $G L_{n}(F)$, Lin. Alg. Appl. 202 (1994), 235-266.

[12] E. M. Sá, Imbedding conditions for $\lambda$-matrices, Linear Algebra and Its Applications 24 (1979), 33-50.

[13] F. C. Silva, Sums and products of matrices with prescribed similarity classes, Linear and Multilinear Algebra 27 (1990), 317-323.

[14] F. C. Silva, The eigenvalues of the product of matrices with prescribed similarity classes, Linear and Multilinear Algebra 34 (1993), 269-277.

[15] A. Sourour, A Factorization Theorem for Matrices, Linear Multilinear 19 (1986), 141-147.

[16] Robert C. Thompson, Invariant factors under rank one perturbations. Canad. J. Math. 32 (1980), no. 1, 240-245.

[17] YuLin Zhang, On the number of invariant polynomials of the product of matrices with prescribed similarity classes, Linear algebra and its Application 277 (1998), 253-269. 